\title{
Blood-brain barrier, reperfusion injury, and hemorrhagic transformation in acute ischemic stroke
}

Rakesh Khatri, MD

Alexander M. McKinney, MD

Barbara Swenson, MD

Vallabh Janardhan, MD

Correspondence \& reprint requests to Dr. Janardhan: drvallabh@yahoo.com

Neurology ${ }^{\circledR}$ 2012;79 (Suppl 1):S52-S57
It has been more than a century since Paul Ehrlich, in 1885, and later his student Edwin Goldmann, proposed that a barrier existed between the CNS and the peripheral circulation. ${ }^{1}$ While studying the limited permeation of potassium ferrocyanate into the brain in 1900, Lewandowsky coined the term bluthirnschranke, blood-brain barrier (BBB). ${ }^{1}$ The $\mathrm{BBB}$ is considered the gatekeeper of the CNS, whose main role is to maintain the fragile homeostasis of the brain designed by segregating the CNS from the systemic circulation.

FUNCTIONAL ANATOMY OF THE BLOODBRAIN BARRIER The BBB is composed of endothelial cells, pericytes, astrocytes, neurons, and the extracellular matrix (ECM), which are collectively known as the neurovascular unit (NVU). BBB endothelial cells lack fenestrations, have tight junctions (TJs), have minimal pinocytotic activity, and express a number of enzymes capable of degrading both harmful and therapeutic molecules. They also have increased mitochondrial content, which is required for the multiple energy-dependent processes involved in nutrient support and protection of the brain. ${ }^{1-3}$ Pericytes are vascular smooth-muscle-lineage cells that occur as solitary cells embedded in the basement membrane of microvessels and have their own characteristic morphology. ${ }^{4}$ Both the endothelial cells and pericytes are surrounded by the basal lamina, which is $30-$ to $40-\mathrm{nm}$ contiguous with the plasma membranes of astrocyte end-feet. The basal lamina supports and anchors cells via adhesion receptors and regulates intercellular communication. Astrocytes play a very important role not only in BBB support and its maintenance but also in neuron-NVU interactions. Under conditions of ischemia, there is a disruption of the bidirectional communication between microvessels and neurons with the participation of the intervening astrocytes. ${ }^{3}$ The greater the distance between the microvessels and the neurons $[(\mathrm{m}-\mathrm{n})$ distance], the higher the likelihood of being prone to ischemic injury. ${ }^{3}$ Microglial cells are basically the macrophages of the CNS and have the potential to release immunoregulatory, inflammatory, and cytotoxic mediators 5 and thereby influence the BBB. ${ }^{6}$ Activation upon insult to the brain causes the "resting" microglia to change from small bodies with longthin processes to a phagocytic form with stubby processes. The ECM is composed of structural proteins, which are susceptible to enzymatic degradation. Endothelial cells, pericytes, and astrocytes express the integrin and dystroglycan families of matrix adhesion receptors, which adhere to the ECM and serve to mediate NVU function. ${ }^{1}$

BBB changes in ischemic stroke, reperfusion injury, and hemorrhagic transformation. Ischemic stroke, reperfusion injury, and hemorrhagic transformation (HT) all involve disruption of the BBB (figure 1). Our understanding of the biochemical pathways, as well as genomic and ultrastructural changes at the level of the BBB, is still evolving.

Impact of ischemia. One of the major events of cerebral ischemia is energy failure due to a lack of glucose and oxygen. Energy failure, in turn, leads to a cascade of events, including depletion of adenosine triphosphate (ATP), a decrease in $\mathrm{Na}^{+}-\mathrm{K}^{+}$ATPase (sodiumpotassium adenosine triphosphatase) activity, a rise in intracellular potassium, lactic acidosis, and release of extracellular glutamate, all of which can result in BBB disruption. $^{7}$

Endothelial swelling may occur within minutes to hours of ischemic onset, leading to narrowing of the internal diameter of the blood vessel. Lactic acidosis also directly contributes to swelling of endothelial cells, neurons, and astrocytes. Experimentally it has been shown that after microsphere-induced cerebral embolism, there is a decrease in occludin and zonula occludens- 1 at the level of the TJs, contributing to increases in paracellular permeability. ${ }^{8}$ Furthermore, induction of proteases (i.e., tissue plasminogen activator [tPA], matrix metalloproteinases [MMPs], cathepsins, and heparanases) contributes to BBB ECM degradation. Such enzyme induction may further perpetuate BBB TJ permeability and anoikis (i.e., apoptosis induced 
Figure 1 Schematic representation of blood-brain barrier (BBB) changes in acute ischemic stroke

\section{Ischemia}

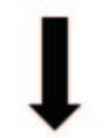

Reperfusion
BBB disruption

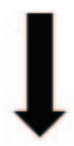

Reperfusion injury
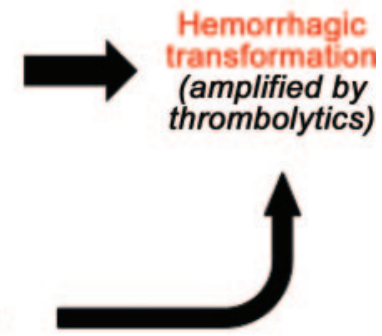

Thrombolytics (IV as well as intra-arterial) can amplify the risk of hemorrhagic transformation secondary to reperfusion injury.

by detachment of cells from the ECM) through integrin-mediated mechanisms. ${ }^{9}$

Microvessel and inflammatory MMP-9 responses have been associated with basal lamina type IV collagen degradation and blood extravasation. ${ }^{3}$ Even though astrocytes are more resistant to glucose deprivation than neurons, the ischemic environment causes endothelial cells to lose contact with the astrocyte feet. ${ }^{10}$

Adding to further insult is nitric oxide (NO), a free radical and neurotoxin with powerful vasodilatory properties that can form reactive oxide species and can exacerbate DNA damage and endothelial injury. The astrocytes themselves express NO synthase during cerebral ischemia, which in turn contributes to peroxynitrate formation and BBB breakdown. ${ }^{7}$

Microvascular leukocyte accumulation has been shown to occur as early as 30 minutes after permanent middle cerebral artery (MCA) occlusion. ${ }^{11}$ Even at the genomic level, changes start to occur within minutes of arterial occlusion. There is an increased expression of early response genes (e.g., c-jun, c-fos), and hours later this is followed by an increase in heat-shock genes (e.g., Hsp70, Hsp72). These genes may have a role in neural plasticity and may serve as an important target for future therapy. ${ }^{11}$

Within the ischemic penumbra, apoptotic pathways are induced through both caspase-dependent (ATP-dependent) and caspase-independent mechanisms. ${ }^{12}$ Proinflammatory cytokines such as interleukin- 1 and tissue necrosis factor- $\alpha$ are induced and are followed by chemokines, which are associated with an activated endothelium (e.g., monocyte chemoattractant protein-1, cytokineinduced neutrophil chemoattractant). ${ }^{12}$ This leads to leukocyte recruitment and extravasation, thereby further enhancing inflammatory activity and toxic free

radical production. ${ }^{12}$ Therefore, the various previously discussed mechanisms initiated during the ischemic cascade have a significant impact on the BBB. ${ }^{12}$

Impact of reperfusion. Reperfusion is essential for brain tissue survival; it also contributes to additional tissue damage and has the potential for HT. ${ }^{12}$ Reperfusion injury has been defined in numerous ways, including activation of endothelium, excess production of oxygen free radicals, inflammatory responses and leukocyte recruitment, increase in cytokine production, and edema formation. Common among these mechanisms is $\mathrm{BBB}$ disruption. ${ }^{13}$

Depending upon the duration and severity of ischemia, degree of reperfusion, and type of stroke animal model, it is proposed that there are 3 stages of paracellular permeability after reperfusion. Stage 1 is reactive hyperemia, which constitutes a loss of cerebral autoregulation, increased BBB permeability, and acute elevation in regional cerebral blood flow. Stage 2 is hypoperfusion (no-reflow effect), which occurs immediately after the stage of hyperemia and is attributed to continued cerebral metabolic depression, microvascular obstruction, occlusion via swelling of endothelial cells and astrocytic end-feet, and formation of endothelial microvilli. This causes nutritional deficiency in brain tissue and enhances neutrophil adhesion, with subsequent inflammatory activity. These events directly contribute to stage 3 , increased paracellular permeability, which occurs as a biphasic response. The first phase occurs 3 to 8 hours postreperfusion and is attributed to increased inflammatory and oxidative stress on the BBB, along with enzymatic degradation of the ECM. The second phase occurs 18 to 96 hours post-reperfusion and coincides with the increased vasogenic edema and angiogenesis. Compared with stage 1 , hyperemia, which is associated with cytotoxic edema, the biphasic response of stage 3 is associated with vasogenic edema. In contrast to cytotoxic edema, vasogenic edema is associated with alterations in BBB TJs, resulting in increased permeability to macromolecules, allowing movement from intracellular to extracellular spaces. ${ }^{10}$

These 3 stages of reperfusion injury have also been demonstrated by radiologic studies. ${ }^{13,14}$ In an animal study, the apparent diffusion coefficient images were studied in a rat with a transiently occluded MCA, which was reperfused after 30 minutes and followed for at least 24 hours. ${ }^{14}$ The apparent diffusion coefficient was found to be decreased by the end of the ischemic event and then improved during the next few hours of reperfusion, only to decrease again at 24 hours, demonstrating secondary ischemic injury. ${ }^{14}$ 

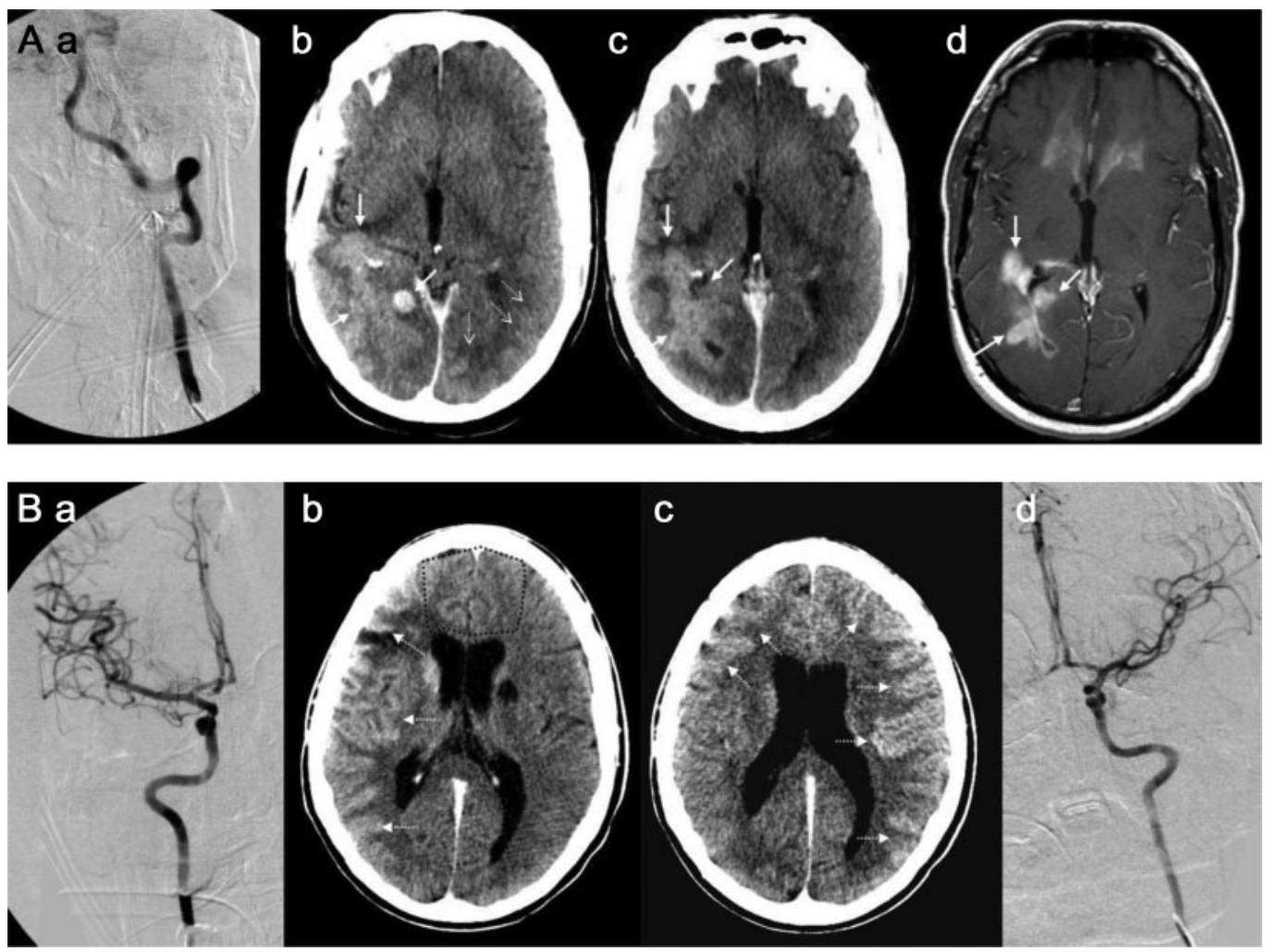

A 43-year-old man (A) with glioblastoma multiforme (GBM) underwent blood-brain barrier disruption (BBBD) via left vertebral artery catheter angiography (a). Within 30 minutes, contrast-enhanced CT was performed, demonstrating enhancement in the cortex of the bilateral posterior cerebral arterial (PCA) distributions (dashed arrows denote normal cortical enhancement on the left side) with enhancing tumor margins surrounding the posterior horn of the right lateral ventricle (arrows, b, c), as noted on postcontrast T1-weighted MRI (d). A 34-year-old man (B) with GBM underwent right internal carotid artery (ICA) catheter BBBD (a), with resultant right middle cerebral arterial (MCA) distribution enhancement (dotted arrows, b) that spares the ACA distributions (dotted outline, c); note the lack of cross-filling into the A1 segment of the right $A C A$, as well as the right MCA, via the anterior communicating artery and the lack of PCA distribution cortical enhancement (a). The next day, the patient underwent catheter BBBD via the left ICA, with demonstration of bilateral ACA distribution cortical enhancement (dotted arrows, c), which may be attributable to the good cross-filling into the bilateral ACAs as well as the $A 1$ segment of the right ACA and right MCA, seen via the left ICA (d).

$B B B$ changes during $H T$. HT is a common and natural consequence of infarction and is likely a multifactorial phenomenon. Structurally, evidence suggests that the fundamental mechanism leading to blood extravasation is disruption of the BBB. Studies have suggested that the cascade related to reperfusion injury and oxidative stress, leukocyte infiltration, vascular activation, and dysregulated extracellular proteolysis acts as a potential trigger of HT by undermining the integrity of both basal lamina and endothelial TJs. ${ }^{15}$ These animal-based proposed mechanisms have also been substantiated in human studies, as evidenced by high MMP-9 levels that independently predicted HT in patients. ${ }^{16}$ Furthermore, free radical production and MMP induction induce the activity of each other.

Clinical implication of BBB disruption. In neurooncology, BBB disruption and convection-enhanced delivery have emerged as leading investigational de- livery techniques for the treatment of malignant brain tumors. ${ }^{17} \mathrm{BBB}$ disruption can be visualized by both head CT and MRI (figures 2 and 3). Similarly, $\mathrm{BBB}$ disruption in acute ischemic stroke has been demonstrated as contrast enhancement on head CT (figure 4) as well as MRI. In a study using brain MRI ${ }^{13}$ the BBB disruption was evident by delayed gadolinium enhancement of CSF space in fluidattenuated inversion recovery images and was termed as hyperintense acute reperfusion marker (HARM). It was demonstrated in 47 (33\%) of 144 patients with ischemic stroke. Reperfusion was found to be the strongest independent predictor of early BBB disruption. HARM has also been associated with HT and worse clinical outcome, but reperfusion was not independently associated with poor outcome.

Both HT and early BBB disruption were more common in patients treated with recombinant tissue plasminogen activator (r-tPA) than those not treated. 


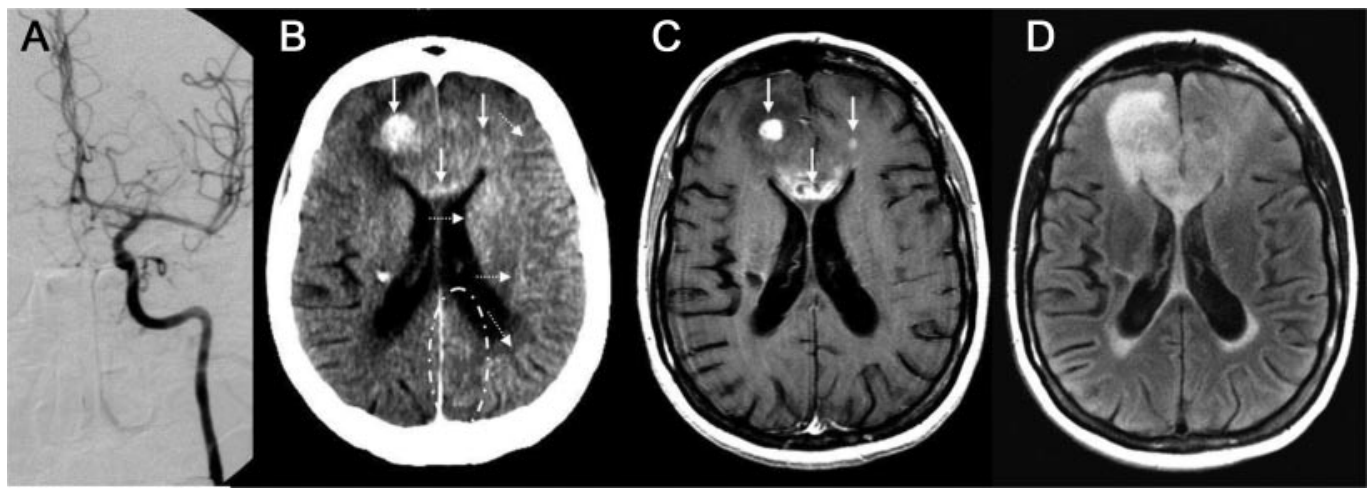

A 39-year-old woman underwent left internal carotid artery (ICA) blood-brain barrier disruption (BBBD) (A), with (as expected) resultant left-sided cortical enhancement on contrast-enhanced CT (CECT) (B, dotted arrows), likely with mild left-sided cortical enhancement in the posterior cerebral arterial (PCA) distribution, as there is a prominent left posterior communicating artery supplying the left PCA (dot-dash outline, B). Postcontrast T1-weighted MRI (C) showed enhancement in foci that correspond to those visualized on the post-BBBD CECT (solid arrows, B, C), with a moderate amount of edema surrounding the right frontal lesion and evidence of spread through the genu of the corpus callosum on fluid-attenuated inversion recovery MRI (D).

The median time estimate of BBB disruption from onset of ischemia was proposed to be 3.8 hours, thus making it important to attempt to reduce complications associated with acute thrombolytic therapy, broadening the therapeutic window and improving clinical outcome. ${ }^{13}$ In a retrospective analysis of MRI performed in ischemic stroke patients, it was suggested that early parenchymal and vascular enhancement of the stroke lesion, suggestive of $\mathrm{BBB}$ disruption, is predictive of symptomatic HT, especially after tPA administration. ${ }^{18}$

Several mechanisms are postulated for contrast extravasation and hemorrhage related to contrast agents. ${ }^{19}$ The most convincing of them includes contrast toxicity on basal lamina, a structural barrier associated with disruption of the BBB. It results in extravasation of cellular blood elements from microvessels that, in turn, causes contrast extravasation (defined as hyperdensity suggestive of contrast [Hounsfield unit >90]) seen at 24 hours (figure 4A). In comparison, contrast enhancement is caused by leakage of contrast medium from vessels into the extracellular spaces, as a result of increased permeability of the BBB. The hyperdensity on head CT secondary to contrast enhancement typically clears within 24 hours (figure 4B). Contrast extravasation has been shown to have a stronger association with $\mathrm{HT}$ as well as a poor prognosis. ${ }^{20}$

Effect of r-tPA on BBB. Recombinant tissue plasminogen activator ( $\mathrm{r}-\mathrm{tPA})$ is an endogenously synthesized extracellular protease and is also a signaling molecule in the brain. It mediates matrix remodeling during brain development and plasticity. HT seen with $\mathrm{r}$-tPA may be an effect of its thrombolytic ac- tion. However, it is also suggested that thrombolytic treatment may exacerbate BBB disruption. It might lead to MMP activation, degradation of the ECM, and alteration of endothelial function. This would promote inflammation by interacting with NMDAtype glutamate receptor and potentially amplify excitotoxic calcium currents. ${ }^{21}$ The risks of HT secondary to reperfusion injury are certainly amplified with the use of IV or intra-arterial thrombolytics vs mechanical thrombectomy alone (figure 1).

Preclinical investigations that involved using pharmacologic strategies to reduce revascularizationinduced HT have provided indirect evidence of the changes discussed above. These strategies included hypothermia, free radical-spin trap compounds, platelet inhibitors, and MMP inhibitors. ${ }^{22}$ In male rats with MCA occlusion, it was suggested that $\mathrm{r}$ tPA-mediated mortality in delayed reperfusion is associated with early opening of the BBB. ${ }^{23}$ Closure of the BBB with MMP inhibitor (BB-94) given before $r$-tPA treatment reduced mortality, suggesting that this treatment might reduce the risk associated with thrombolysis. ${ }^{23}$

NVUs are also niches for neural stem/progenitor cells in the adult brain. Within the NVUs, newly born, immature neurons closely associate with the remodeling vasculature. ${ }^{24}$ Preclinical studies have revealed that brain injury induces neurogenesis (the generation of new neurons) and angiogenesis (the growth of new blood vessels). Agents and manipulations that boost angiogenesis or neurogenesis may promote functional recovery after brain injuries. ${ }^{25}$ This suggests that the manipulation of endogenous neural precursors and endothelial cells may be poten- 
the manuscript, study concept or design, analysis or interpretation of data, study supervision.
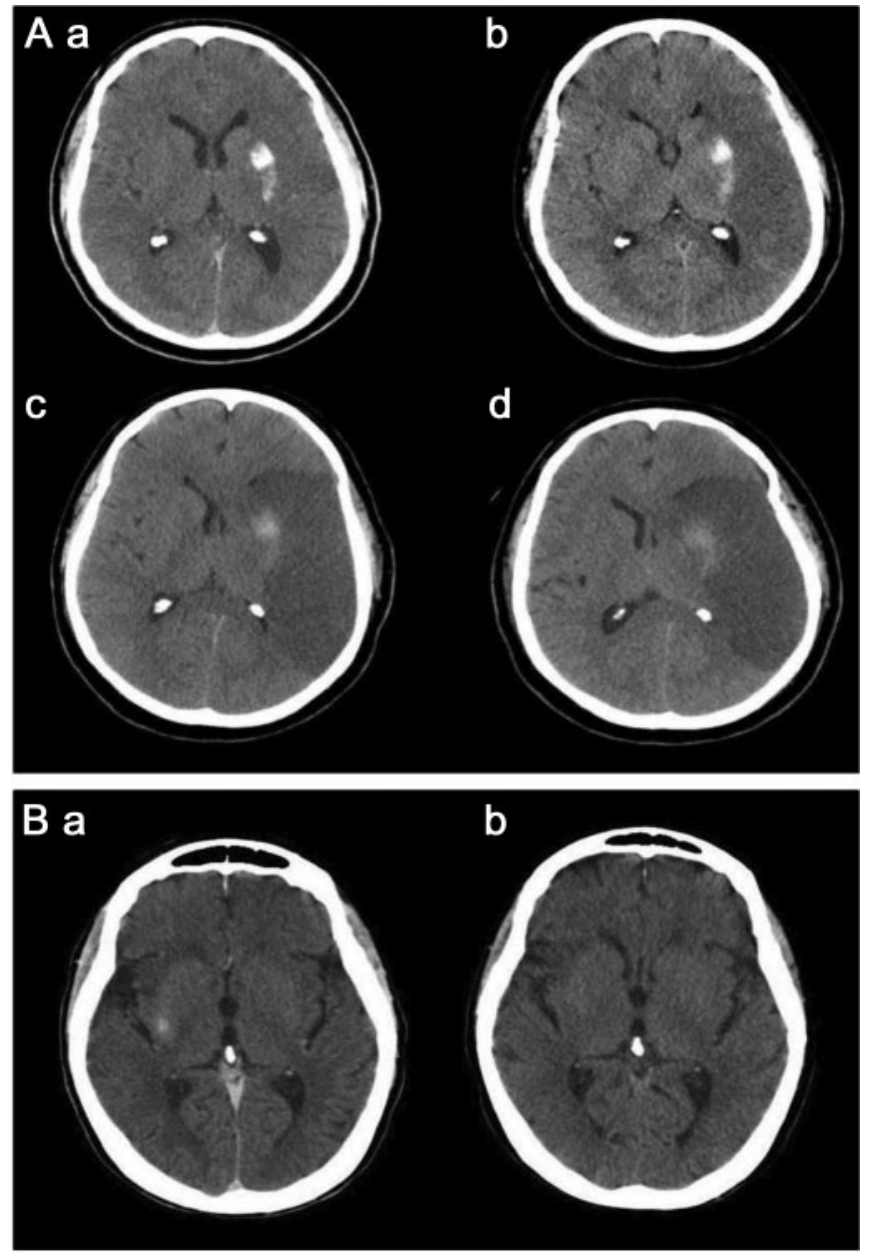

Immediately post intra-arterial thrombolysis head CT (A) shows a new area of hyperdensity within the left lentiform nucleus (a). Fourteen hours postprocedure head CT showed persistent hyperdensity, loss of gray-white differentiation in the left parieto-temporal convexities, and effacement of the left lateral ventricle (b). Follow-up head CT scans at 36 hours and 60 hours showed persistent hyperdensity in the left putamen, suggesting contrast extravasation with increase in cerebral edema and midline shift (c, d). Head CT immediately post intra-arterial thrombolysis (B) shows a hyperdensity in the right putamen (a), which resolved completely on a repeat head CT performed 16 hours postprocedure (b), suggesting contrast enhancement.

\section{DISCLOSURE}

Dr. Khatri, Dr. Swenson, and Dr. Janardhan report no disclosures. Dr. McKinney serves as a Medical Advisory Board member for Vital Images; receives travel reimbursement and hardware for research; has served as Associate Editor of Yearbook of Neurology and Neurosurgery; and spends $5 \%$ effort of clinical time performing blood-brain barrier disruptions. Go to Neurology.org for full disclosures.

Received June 27, 2011. Accepted in final form January 17, 2012.

\section{REFERENCES}

1. Hawkins BT, Davis TP. The blood-brain barrier/neurovascular unit in health and disease. Pharmacol Rev 2005; 57:173-185.

2. Oldendorf WH, Cornford ME, Brown WJ. The large apparent work capability of the blood-brain barrier: a study of the mitochondrial content of capillary endothelial cells in brain and other tissues of the rat. Ann Neurol 1977;1: $409-417$.

3. del Zoppo GJ. The neurovascular unit, matrix proteases, and innate inflammation. Ann NY Acad Sci 2010;1207: 46-49.

4. von Tell D, Armulik A, Betsholtz C. Pericytes and vascular stability. Exp Cell Res 2006;312:623-629.

5. Choi YK, Kim KW. Blood-neural barrier: its diversity and coordinated cell-to-cell communication. BMB Rep 2008; 41:345-352.

6. Zlokovic BV. The blood-brain barrier in health and chronic neurodegenerative disorders. Neuron 2008;57: $178-201$.

7. Kulik T, Kusano Y, Aronhime S, Sandler AL, Winn HR. Regulation of cerebral vasculature in normal and ischemic brain. Neuropharmacology 2008;55:281-288.

8. Kago T, Takagi N, Date I, Takenaga Y, Takagi K, Takeo S. Cerebral ischemia enhances tyrosine phosphorylation of occludin in brain capillaries. Biochem Biophys Res Commun 2006;339:1197-1203.

9. Grossmann J. Molecular mechanisms of "detachmentinduced apoptosis-Anoikis.” Apoptosis 2002;7:247-260.

10. Heo JH, Han SW, Lee SK. Free radicals as triggers of brain edema formation after stroke. Free Radic Biol Med 2005; 39:51-70.

11. Huang WC, Chen JJ, Inoue H, Chen CC. Tyrosine phosphorylation of I-kappa B kinase alpha/beta by protein kinase C-dependent c-Src activation is involved in TNFalpha-induced cyclooxygenase- 2 expression. J Immunol 2003; 170:4767-4775.

12. Janardhan V, Qureshi AI. Mechanisms of ischemic brain injury. Curr Cardiol Rep 2004;6:117-123.

DISCUSSION BBB changes during ischemia and reperfusion are dynamic and complex. An understanding of these BBB changes in acute ischemic stroke could lead to better patient selection as well as safer and potentially improved clinical outcomes with existing therapies such as thrombolysis (IV and intra-arterial) and mechanical thrombectomy.

\section{AUTHOR CONTRIBUTIONS}

Dr. Khatri and Dr. Swenson: drafting/revising the manuscript. Dr. McKinney: drafting/revising the manuscript, analysis or interpretation of data, acquisition of data, study supervision. Dr. Janardhan: drafting/ revising
13. Warach S, Latour LL. Evidence of reperfusion injury, exacerbated by thrombolytic therapy, in human focal brain ischemia using a novel imaging marker of early bloodbrain barrier disruption. Stroke 2004;35:2659-2661.

14. Neumann-Haefelin T, Kastrup A, de Crespigny A, et al. Serial MRI after transient focal cerebral ischemia in rats: dynamics of tissue injury, blood-brain barrier damage, and edema formation. Stroke 2000;31:1965-1973.

15. Wang X, Tsuji K, Lee SR, et al. Mechanisms of hemorrhagic transformation after tissue plasminogen activator reperfusion therapy for ischemic stroke. Stroke 2004;35: $2726-2730$. 
16. Castellanos M, Leira R, Serena J, et al. Plasma metalloproteinase-9 concentration predicts hemorrhagic transformation in acute ischemic stroke. Stroke 2003;34: $40-46$.

17. Bidros DS, Vogelbaum MA. Novel drug delivery strategies in neuro-oncology. Neurotherapeutics 2009;6:539-546.

18. Vo KD, Santiago F, Lin W, Hsu CY, Lee Y, Lee JM. MR imaging enhancement patterns as predictors of hemorrhagic transformation in acute ischemic stroke. AJNR Am J Neuroradiol 2003;24:674-679.

19. Yoon W, Seo JJ, Kim JK, Cho KH, Park JG, Kang HK. Contrast enhancement and contrast extravasation on computed tomography after intra-arterial thrombolysis in patients with acute ischemic stroke. Stroke 2004;35:876881.

20. Kim J, Smith A, Hemphill JC 3rd, et al. Contrast extravasation on $\mathrm{CT}$ predicts mortality in primary intracerebral hemorrhage. AJNR Am J Neuroradiol 2008;29:520-525.
21. Wang X, Lo EH. Triggers and mediators of hemorrhagic transformation in cerebral ischemia. Mol Neurobiol 2003; 28:229-244.

22. Khatri P, Wechsler LR, Broderick JP. Intracranial hemorrhage associated with revascularization therapies. Stroke 2007;38:431-440.

23. Pfefferkorn T, Rosenberg GA. Closure of the blood-brain barrier by matrix metalloproteinase inhibition reduces rtPA-mediated mortality in cerebral ischemia with delayed reperfusion. Stroke 2003;34:2025-2030.

24. Xiong Y, Mahmood A, Chopp M. Angiogenesis, neurogenesis and brain recovery of function following injury. Curr Opin Investig Drugs 2010;11:298-308.

25. Zhang ZG, Chopp M. Neurorestorative therapies for stroke: underlying mechanisms and translation to the clinic. Lancet Neurol 2009;8:491-500.

26. Beck H, Plate KH. Angiogenesis after cerebral ischemia. Acta Neuropathol 2009;117:481-496. 


\section{Neurology}

\section{Blood-brain barrier, reperfusion injury, and hemorrhagic transformation in acute ischemic stroke}

Rakesh Khatri, Alexander M. McKinney, Barbara Swenson, et al. Neurology 2012;79;:S52-S57

DOI 10.1212/WNL.0b013e3182697e70

This information is current as of September 24, 2012

\section{Updated Information \&} Services

References

Citations

Permissions \& Licensing

Reprints including high resolution figures, can be found at: http://n.neurology.org/content/79/13_Supplement_1/S52.full

This article cites 26 articles, 11 of which you can access for free at: http://n.neurology.org/content/79/13_Supplement_1/S52.full\#ref-list-1

This article has been cited by 7 HighWire-hosted articles: http://n.neurology.org/content/79/13_Supplement_1/S52.full\#\#otherarti cles

Information about reproducing this article in parts (figures,tables) or in its entirety can be found online at:

http://www.neurology.org/about/about_the_journal\#permissions

Information about ordering reprints can be found online:

http://n.neurology.org/subscribers/advertise

Neurology ${ }^{\circledR}$ is the official journal of the American Academy of Neurology. Published continuously since 1951, it is now a weekly with 48 issues per year. Copyright Copyright $@ 2012$ by AAN Enterprises, Inc.. All rights reserved. Print ISSN: 0028-3878. Online ISSN: 1526-632X.

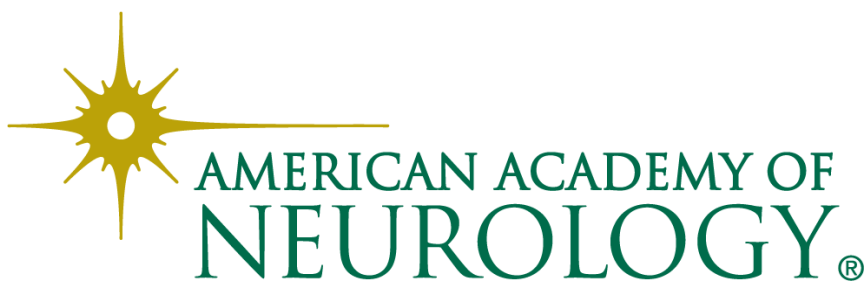

